\title{
Prevalence of antimicrobial resistance in enteric Escherichia coli from domestic pets and assessment of associated risk markers using a generalized linear mixed model
}

\author{
Liliana R. Leite-Martins ${ }^{a, *}$, Maria I.M. Mahú ${ }^{\mathrm{b}}$, Ana L. Costa $^{\mathrm{b}}$, Ângelo Mendes $^{\mathrm{b}}$, \\ Elisabete Lopes ${ }^{\mathrm{b}}$, Denisa M.V. Mendonça ${ }^{\mathrm{c}, \mathrm{d}}$, João J.R. Niza-Ribeiro ${ }^{\mathrm{c}, \mathrm{d}}$, \\ Augusto J.F. de Matos ${ }^{\mathrm{a}}$, Paulo Martins da Costa ${ }^{\mathrm{b}}$ \\ a Veterinary Clinics Department, Abel Salazar Institute for the Biomedical Sciences (ICBAS), Porto University (UP), Portugal \\ b Microbiology and Food Technology Department, Abel Salazar Institute for the Biomedical Sciences (ICBAS), Porto University (UP), \\ Portugal \\ c Population Studies Department, Abel Salazar Institute for the Biomedical Sciences (ICBAS), Porto University (UP), Portugal \\ d Public Health Institute (ISPUP), Porto University (UP), Portugal
}

\section{A R T I C L E I N F O}

\section{Article history:}

Received 3 February 2014

Received in revised form

19 September 2014

Accepted 19 September 2014

\section{Keywords:}

Antimicrobial resistance

Pets

Escherichia coli

Prevalence

Risk markers

\begin{abstract}
A B S T R A C T
Antimicrobial resistance (AMR) is a growing global public health problem, which is caused by the use of antimicrobials in both human and animal medical practice. The objectives of the present cross-sectional study were as follows: (1) to determine the prevalence of resistance in Escherichia coli isolated from the feces of pets from the Porto region of Portugal against 19 antimicrobial agents and (2) to assess the individual, clinical and environmental characteristics associated with each pet as risk markers for the AMR of the E. coli isolates.

From September 2009 to May 2012, rectal swabs were collected from pets selected using a systematic random procedure from the ordinary population of animals attending the Veterinary Hospital of Porto University. A total of 78 dogs and 22 cats were sampled with the objective of isolating E. coli. The animals' owners, who allowed the collection of fecal samples from their pets, answered a questionnaire to collect information about the markers that could influence the AMR of the enteric E. coli. Chromocult tryptone bile X-glucuronide agar was used for E. coli isolation, and the disk diffusion method was used to determine the antimicrobial susceptibility. The data were analyzed using a multilevel, univariable and multivariable generalized linear mixed model (GLMM). Several (49.7\%) of the 396 isolates obtained in this study were multidrug-resistant. The E. coli isolates exhibited resistance to the antimicrobial agent's ampicillin (51.3\%), cephalothin (46.7\%), tetracycline (45.2\%) and streptomycin (43.4\%). Previous quinolone treatment was the main risk marker for the presence of AMR for 12 (ampicillin, cephalothin, ceftazidime, cefotaxime, nalidixic acid, ciprofloxacin, gentamicin, tetracycline, streptomycin, chloramphenicol, trimethoprim-sulfamethoxazole and aztreonam) of the 15 antimicrobials assessed. Coprophagic habits were also positively associated with an increased risk of AMR for six drugs, ampicillin, amoxicillin-clavulanic acid, cephamycin, ciprofloxacin, streptomycin, and trimethoprim-sulfamethoxazole.
\end{abstract}

\footnotetext{
* Corresponding author at: UPVET - Clínica Veterinária da Universidade do Porto, Departamento de Clínicas Veterinárias, Instituto de Ciências Biomédicas Abel Salazar, Universidade do Porto, Rua Jorge Viterbo Ferreira, 228, 4050-313 Porto, Portugal. Tel.: +351 220428000 ; fax: +351 226093390.

E-mail address: Imartins@icbas.up.pt (L.R. Leite-Martins).
} 
In summary, pets with a record of one or more previous quinolone treatments and exhibiting coprophagic habits were at an increased risk of harboring multidrug-resistant $E$. coli strains in their feces compared to pets without these characteristics. AMR is a serious global problem, and assessing the risk markers for the presence of drug-resistant bacteria in pets, a very close source of resistance determinants to humans, is essential for the implementation of safe handling procedures for companion animals and for the prudent selection of antimicrobial compounds in veterinary practice.

(c) 2014 Elsevier B.V. All rights reserved.

\section{Introduction}

Antimicrobial resistance (AMR) is one of the primary global public health problems of the next decade (Carlet et al., 2012). The main driving force of AMR, which is based on the genetic plasticity of bacteria, is the selective pressure exerted by antimicrobial usage in human and veterinary medicine, animal and fish production, and agricultural and food technology (Kearns, 2010; EAAD, 2013; Martins da Costa et al., 2013). Resistant bacteria may be transmitted between interdependent hosts and spread into the environment, contributing to the worldwide increase of AMR (CDC, 2013). The progress in veterinary medicine and the number of domestic pets treated by specialized practitioners has increased the use of antimicrobial treatments (Martins da Costa et al., 2013). Additionally, pets live longer and are in closer contact with their owners, favoring the mutual transfer of microbial flora, either directly by contact with skin or bacteria-containing material, such as saliva and feces, or indirectly via the household environment (Martins et al., 2013). When introduced to a new host, the resistant bacteria can colonize, infect, or remain in that particular environment for very short periods of time. In all cases, resistant bacteria can either spread their resistance genes to host-resident bacteria, either commensals or pathogenic, or accept resistance genes from such microorganisms (Jernberg et al., 2010). As a consequence, AMR in companion animals is simultaneously an important veterinary medical issue and a public health concern (Lloyd, 2007).

The regular monitoring of AMR in pathogenic and normal flora has been recommended by the World Health Organization and the European Centre for Disease Prevention and Control. For this purpose, the European Antimicrobial Resistance Surveillance Network (EARSNet), involving 53 countries, was created (EFSA and ECDPC, 2013). Similar programs have been proposed for veterinary medicine, leading to field studies of food animals (Aarestrup, 2004; Taylor et al., 2008) and pets (Moyaert et al., 2006; Lloyd, 2007; Costa et al., 2008; Murphy et al., 2009; Leonard et al., 2012). However, to the best of our knowledge, no studies have included the clinical histories of both pets and their cohabitants and household features to assess potential AMR risk markers.

Escherichia coli is an important member of the normal intestinal microflora of humans and other mammals, but it is also a highly versatile pathogen, causing diverse intestinal and extra intestinal diseases via virulence factors that affect a wide range of cellular processes (Kaper et al., 2004). AMR has been associated with several treatment failures in both human and veterinary patients (Toutain et al., 2010; Vigil et al., 2009).

The objective of the present study was to determine the proportion of antimicrobial-resistant $E$. coli isolated from the feces of pets from the Porto region of Portugal and to assess the individual, clinical and environmental characteristics of pets as risk markers for the AMR in the isolated strains. It is hypothesized that animals with a relevant clinical background will harbor more resistant E. coli isolates.

\section{Materials and methods}

\subsection{Enrollment and sampling}

Only dogs and cats were enrolled in the study. A random systematic approach was used to select animals for the present cross-sectional study, which was performed at the Veterinary Hospital of Porto University (UPVET).

From September 2009 to May 2012, on Monday or Tuesday, one of the first five pets to arrive at the UPVET attending room was randomly selected for inclusion in the study. If the owners refused to participate in the study, the next pet, in order of arrival, was included. To be eligible for enrollment in the study, the animal should not have received any antimicrobial therapy within the preceding 4 months. The owners were asked to sign a consent form, fill out a questionnaire and allow the collection of fecal samples from their pets using rectal swabs. The Ethics Committee of the Abel Salazar Institute for the Biomedical Sciences, University of Porto approved this study.

\subsection{Questionnaire}

The owners were asked to fill out a brief questionnaire to provide information about the possible risk markers for multidrug-resistant E. coli. Multidrug resistance was defined according to Magiorakos et al. (2012). The questionnaire was conducted by the first author and constructed following similar studies in animals (Akwar et al., 2007; Ahmed et al., 2012; Boothe, 2012) and humans (McDonald et al., 2001; Sotto et al., 2001; Lietzau et al., 2007; Kalter et al., 2010; Lastours et al., 2010). To evaluate the potential risk markers, the questionnaires included the following individual and clinical characteristics: (1) species, (2) gender, (3) age, (4) daily access to the outside environment (indoor habitat refers to those animals that live predominantly at home or with very restricted outdoor access), (5) diet (commercial refers to the animals that were fed strictly commercial dry or wet foods), (6) 
coprophagic habits (ingestion of feces, both their own or from other animals), (7) previous systemic antimicrobial treatments with particular emphasis on (8) previous systemic quinolone treatments (assessed through the clinical file of the pet),(9) existence of cohabitant pets in the household, (10) previous antimicrobial treatments received by the owners, (11) owner's professional connection with healthcare units, such as human or veterinary hospitals, clinics or health centers (such owners were classified as Health Professionals), and (12) reason for veterinary visit (recorded by the veterinary surgeon following a complete physical examination).

\subsection{E. coli isolation}

Fecal samples were obtained using saline wet swabs that were introduced with circular movements into the rectum of each animal. The swabs were immediately immersed in buffered peptone water (BPW) (Oxoid, Basingstoke, Hampshire, England), transported to the laboratory and stored at room temperature for $1 \mathrm{~h}$. Then, for $E$. coli isolation, an aliquot of $5 \mu \mathrm{L}$ was streaked onto Chromocult tryptone bile X-glucuronide (TBX) agar (Biokar Diagnostics, Allonne, Beauvais, France) and incubated at $37^{\circ} \mathrm{C}$ for $24 \mathrm{~h}$. Two to five confirmed pure colonies with a typical appearance of $E$. coli were selected on the basis of colony size and morphology. The described procedure and the biochemical confirmation of the isolates were adapted from standard protocols used in similar studies to achieve the most reliable and accurate $E$. coli detection (Costa et al., 2008; Simões et al., 2010; Martins et al., 2013).

\subsection{Antimicrobial susceptibility characterization}

A disk diffusion assay following the standard guidelines (CLSI, 2012) was performed to assess the antimicrobial susceptibility of each isolate. The antimicrobial drugs were selected to include those regularly used in both human and veterinary medicine and to represent different antimicrobial classes (Goossens et al., 2005; Elseviers et al., 2007; EFSA and ECDPC, 2013). A total of 19 antimicrobial agents (AM) (Oxoid, Basingstoke, Hampshire, England) were used: ampicillin (AMP, $10 \mu \mathrm{g}$ ), amoxicillin-clavulanic acid (AMC, $30 \mu \mathrm{g}$ ), cephalothin (CEF, $30 \mu \mathrm{g}$ ), cefoxitin (FOX, $30 \mu \mathrm{g})$, ceftazidime (CAZ, $30 \mu \mathrm{g})$, cefotaxime (CTX, $30 \mu \mathrm{g}$ ), nalidixic acid (NAL, $30 \mu \mathrm{g}$ ), ciprofloxacin (CIP, $5 \mu \mathrm{g}$ ), gentamicin (GEN, $10 \mu \mathrm{g}$ ), tetracycline (TET, $30 \mu \mathrm{g}$ ), streptomycin (STR, $10 \mu \mathrm{g})$, amikacin (AMK, $30 \mu \mathrm{g}$ ), trimethoprim-sulfamethoxazole (SXT, $25 \mu \mathrm{g}$ ), chloramphenicol (CHL, $30 \mu \mathrm{g}$ ), tobramycin (TOB, $10 \mu \mathrm{g}$ ), kanamycin (KAN, $30 \mu \mathrm{g}$ ), aztreonam (ATM, $30 \mu \mathrm{g}$ ), imipenem (IPM, $10 \mu \mathrm{g}$ ), and nitrofurantoin (NIT, $300 \mu \mathrm{g}$ ). The interpretation of the inhibition zone length was based on the recommendations of the Clinical and Laboratory Standards Institute (CLSI) and breakpoints for Enterobacteriaceae (CLSI, 2012).

\subsection{Data analysis}

The prevalence of AMR for each AM was calculated by dividing the number of resistant $E$. coli isolates by the total number of $E$. coli tested. The potential risk markers obtained from the questionnaire were analyzed as categorical variables as follows: dichotomous variables, such as species (canine, feline), gender (male, female), reason for veterinary visit (routine check-up, illness signs), habitat type (indoor, mixed), diet (commercial, mixed), previous quinolone treatments (yes, no), health professional owners (yes, no), owners administered previous antimicrobial treatments (yes, no), cohabitant pets (yes, no), coprophagy habits (yes, no), and exposure of the animal to any previous antimicrobial treatment, which was transformed into a categorical variable with three levels: "none", "just one", and "two or more". Age was also categorized using three levels as follows: "young" (less than 2 years of age), "adult" (between 2 and 10 years of age), and "old" (greater than 10 years of age). The outcome of the analysis was the result of the AMR, which was dichotomized as either resistant or sensitive. The intermediate results were categorized as sensitive. Using the European Food Safety Authority criteria, each antimicrobial was further classified into one of the following categories for the prevalence of AMR: extremely high, >70\%; very high, 50-70\%; high, 20-50\%; moderate, $10-20 \%$; low, $1-10 \%$; very low, $0.1-1 \%$; and rare, $<0.1 \%$ (EFSA and ECDPC, 2013).

A descriptive analysis of AMR prevalence and the risk markers distribution among $E$. coli isolates was performed (Table 4). To assess the strength of associations between the suggested risk markers and each $\operatorname{AM}(n=15)$, multilevel generalized linear mixed models (GLMM) were developed. The logit link function was used to model the probability of occurrence of resistance to an antibiotic. For the multilevel structure of the data, i.e., more than one E. coli strain (i) was isolated from each animal $(j)$, a two-level structure, in which the $E$. coli strains (first level) were nested within the animal from which they were isolated (second level), was assumed.

The data were modeled as follows:

$Y=\left\{\begin{array}{c}0(\text { no } A M R) \\ 1(A M R)\end{array}\right.$ where $Y$ is the response variable.Pr $(Y)=p_{i j}, i=1, \ldots, 396$ and $j=1, \ldots, 100$.

The generic model used the following equation:

$\operatorname{logit}\left(p_{i j}\right)=a+c_{j}+\beta$ animal variables $j$

In the model, the animal (pet) was allowed to be random. The second-level random effect was given by $\mathrm{c}_{j} \sim N(0$, $\sigma^{2}$ ), where $\sigma^{2}$ is the variance of the random effects at the animal level.

The basic multivariable multilevel model was as follows:

$$
\begin{aligned}
\operatorname{logit}\left(p_{i j}\right)= & a+c_{j}+\beta_{1} \text { Species }_{j}+\beta_{2} \text { Age }_{j}+\beta_{3} \text { Gender }_{j} \\
& +\beta_{4} \text { Reason for visit }_{j}+\beta_{5} \text { Habitat }_{j}+\beta_{6} \text { Diet }_{j} \\
& +\beta_{7} \text { Number AM treatments }_{j} \beta_{8}
\end{aligned}
$$

Previous Qutnolone treatments

$+\beta_{9}$ Owner's profession $_{j} \mid \beta_{10}$

Owner's AM treatments ${ }_{j} \mid \beta_{11}$

Cohabitant pets $_{j}+\beta_{12}$ Coprophagy habits $_{j}$ 
A three-step procedure was performed. Firstly, for each of the evaluated antimicrobials, an univariable multilevel GLMM analysis was conducted to assess the individual relationship between each potential risk factor and the presence of AMR. The second step involved a multivariable multilevel GLMM analysis with all of the variables with $p<0.15$ in the previous analyses. In the third step, a manual backwards and forwards procedure was used to obtain a final model in which each factor effect was adjusted for the remaining factors. Only factors with $p<0.05$ were retained in the final model. Two way interactions were tested for the factors retained in each final model. The data were analyzed using the GEE procedure in the SPSS Software V. 21.0 (IBM SPSS statistical 21 package, IBM Corporation, NY, USA). Interactions were tested and found to be not significant.

The procedure explained above was applied to the 15 AM resulting in 15 different final models, although based in the same general model.

\section{Results}

A total of 78 dogs and 22 cats from 100 distinct households were enrolled. Overall, $396 \mathrm{E}$. coli isolates were obtained, 307 (77.5\%) isolated from dogs and 89 (22.5\%) isolated from cats. Between two and five isolates were obtained per pet with a median of 2 isolates per animal.

\subsection{Antimicrobial resistance profiles}

Our results showed that $28.8 \%$ of the isolates were susceptible to all of the tested compounds. It was also observed that $50 \%$ of the isolates (median) had up to three antimicrobial resistances; finally, it was noticed that $75 \%$ (third quartile) of the isolates were resistant to at most seven drugs. Extreme resistance toward 14 or 15 AMs was observed in five isolates (1.3\%) (data not shown). The histogram displaying the absolute number antimicrobials to which the isolates were resistant suggests the existence of two or possibly three subpopulations of E. coli (Fig. 1): one group was resistant to at most four antimicrobials, the second group was resistant to 5-10 antimicrobials, and a possible third group with isolates having more than 10 antimicrobial resistances.

\subsection{Prevalence of antimicrobial resistance}

The prevalence of AMR varied from $0 \%$ for nitrofurantoin and imipenem to $51.3 \%( \pm 0.049)$ for ampicillin. After categorization according to the EFSA (EFSA and ECDPC, 2013) recommendations, 5.3\% $( \pm 0.022)$ of the tested AMs were in the very high resistance category, $31.6 \%( \pm 0.046)$ were in the high resistance group, and a similar proportion were in the moderate resistance category, as shown in Table 1.

\subsection{Distribution of potential risk markers associated with pets}

The frequency of each tested potential risk marker is shown in Table 2. After looking at the factors species, age, sex and reason for visit it was concluded that the population of pets enrolled in our study resembled fairly the population of cats and dogs attending the hospital.

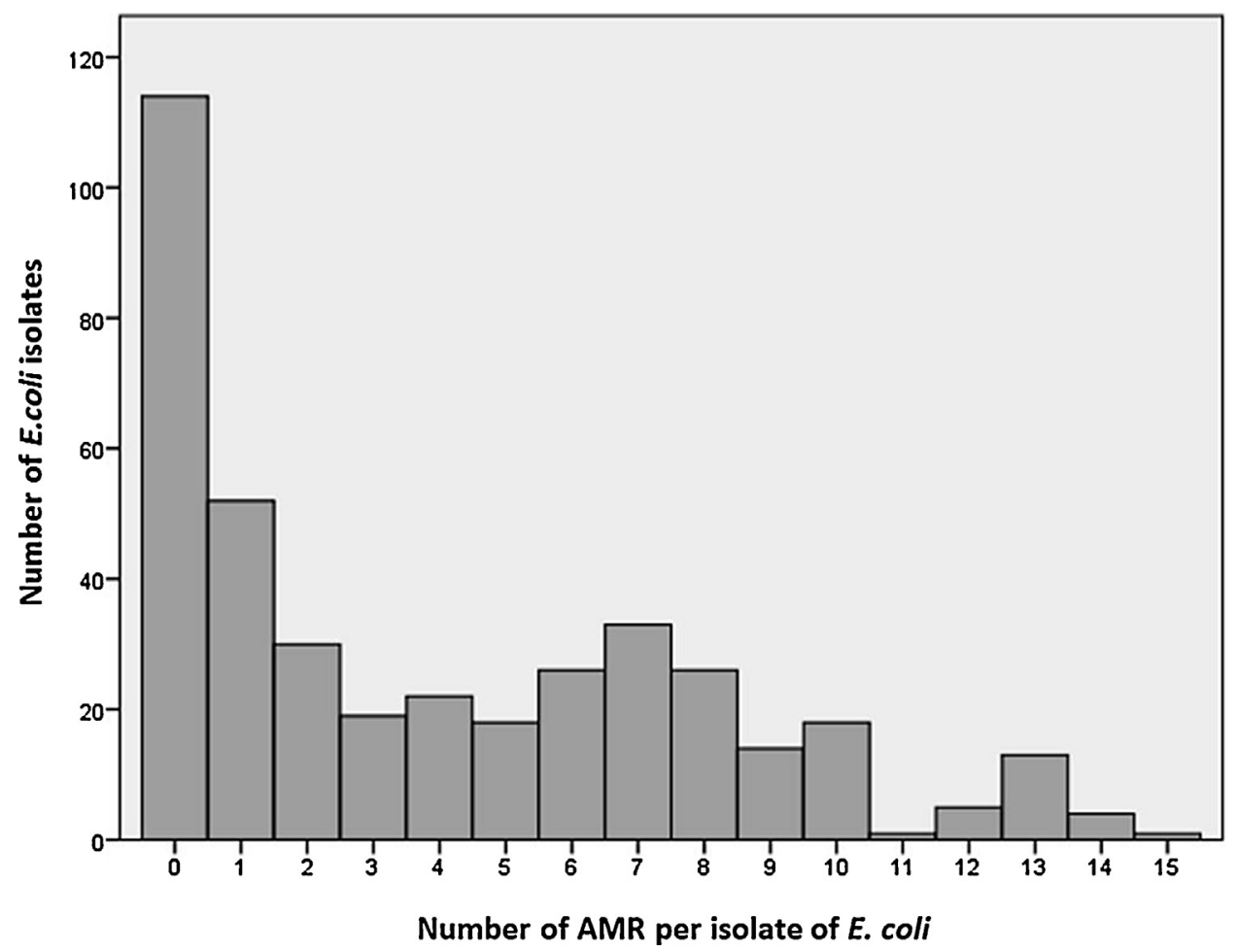

Fig. 1. Frequency of antimicrobial resistance in Escherichia coli isolates $(n=396)$. 
Table 1

Categorization of the antimicrobials (AM) tested with Escherichia coli isolates $(n=396)$ according to the EFSA risk categories for the prevalence of resistance.

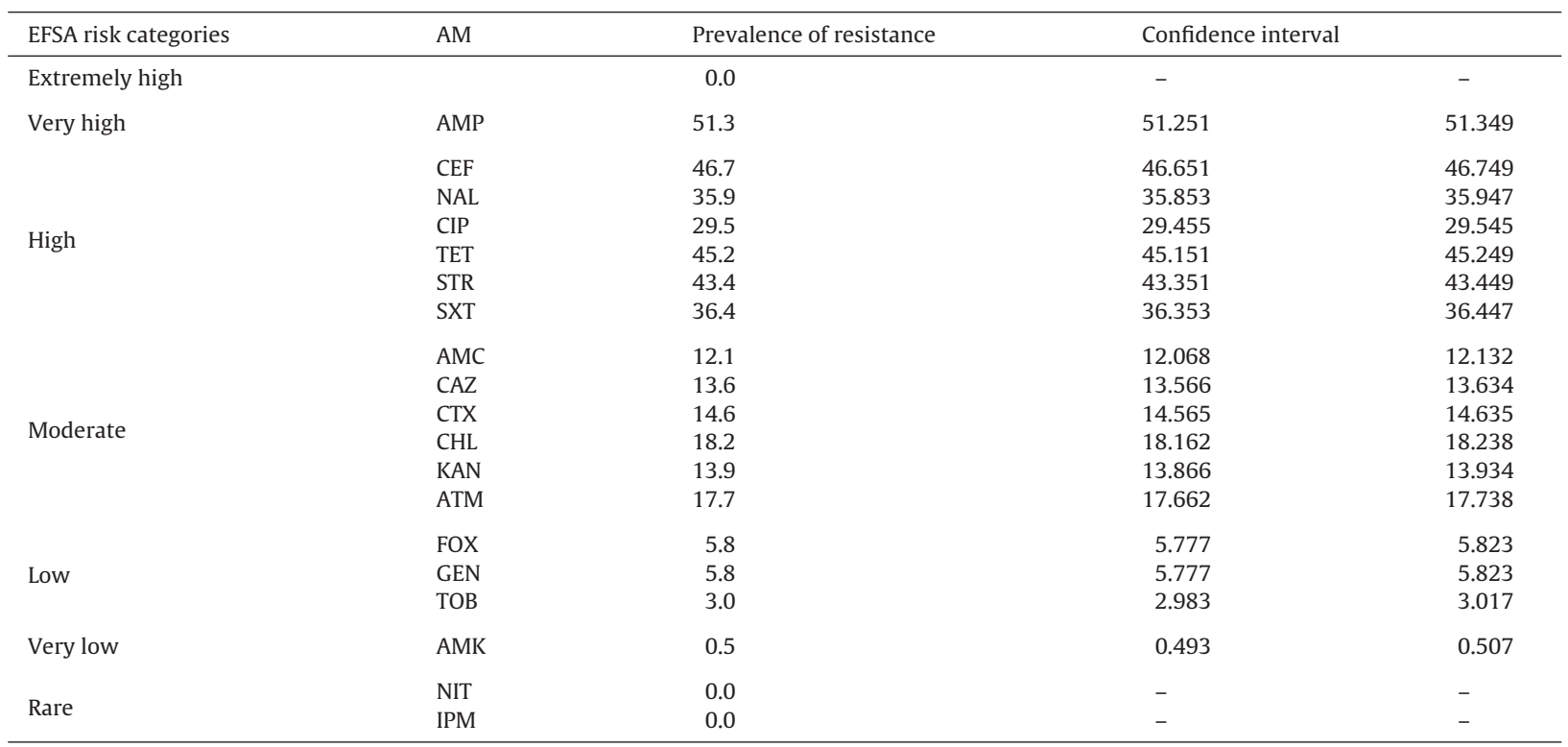

Legend: AM - antimicrobial agent; C.I. - Confidence interval; AMP - ampicillin; AMC - amoxicillin-clavulanic acid; CEF - cephalothin; FOX - cephoxitin; CAZ - ceftazidime; CTX - cefotaxime; NAL - nalidixic acid; CIP - ciprofloxacin; GEN - gentamicin; NIT - nitrofurantoin; TET - tetracycline; STR - streptomycin; AMK - amikacin; SXT - trimethoprim-sulfamethoxazole; CHL - chloramphenicol; TOB - tobramycin; KAN - kanamycin; IPM - imipenem; ATM - aztreonam. The values are expressed in percentages.

Table 2

Distribution of the potential risk marker categories among pets $(n=100)$.

\begin{tabular}{|c|c|c|c|c|c|c|}
\hline Risk marker & Category & Dogs & $\%$ Dogs & Cats & $\%$ Cats & Dogs + cats \\
\hline Species & & 78 & & 22 & 100 & \\
\hline \multirow[t]{3}{*}{ Age } & $<2$ years & 24 & 30 & 9 & 40 & 33 \\
\hline & $2-10$ years & 34 & 43 & 11 & 50 & 45 \\
\hline & $>10$ years & 20 & 25 & 2 & 9 & 22 \\
\hline \multirow[t]{2}{*}{ Gender } & Female & 47 & 60 & 9 & 40 & 56 \\
\hline & Male & 31 & 39 & 13 & 59 & 44 \\
\hline \multirow[t]{2}{*}{ Reason for veterinary visit } & Check up & 23 & 29 & 15 & 68 & 38 \\
\hline & Illness & 55 & 70 & 7 & 31 & 62 \\
\hline \multirow[t]{2}{*}{ Habitat type } & Indoor & 9 & 11 & 15 & 68 & 24 \\
\hline & Mixed & 69 & 88 & 7 & 31 & 76 \\
\hline \multirow[t]{2}{*}{ Diet } & Commercial & 25 & 32 & 10 & 45 & 35 \\
\hline & Mixed & 53 & 67 & 12 & 54 & 65 \\
\hline \multirow[t]{3}{*}{ Animal antimicrobial treatments } & None & 21 & 26 & 17 & 77 & 38 \\
\hline & Just one & 19 & 24 & 3 & 13 & 22 \\
\hline & Two/more & 38 & 48 & 2 & 9 & 40 \\
\hline \multirow[t]{2}{*}{ Animal quinolone treatments } & Yes & 28 & 35 & 2 & 9 & 30 \\
\hline & No & 50 & 64 & 20 & 90 & 70 \\
\hline \multirow[t]{2}{*}{ Owners health professionals } & Yes & 16 & 20 & 6 & 27 & 22 \\
\hline & No & 62 & 79 & 16 & 72 & 78 \\
\hline \multirow[t]{2}{*}{ Owners antimicrobial treatments } & Yes & 38 & 48 & 6 & 27 & 44 \\
\hline & No & 40 & 51 & 16 & 72 & 56 \\
\hline \multirow[t]{2}{*}{ Cohabitant pets } & Yes & 36 & 46 & 16 & 72 & 52 \\
\hline & No & 42 & 53 & 6 & 27 & 48 \\
\hline \multirow[t]{2}{*}{ Coprophagy habits } & Yes & 29 & 37 & 4 & 18 & 33 \\
\hline & No & 49 & 62 & 18 & 81 & 67 \\
\hline
\end{tabular}


Table 3

Distribution of the Escherichia coli isolates $(n=396)$ of canine $(n=307)$ and feline $(n=89)$ origin by potential risk marker categories.

\begin{tabular}{|c|c|c|c|c|}
\hline Risk marker & Category & Canine isolates & Feline isolates & Total isolates (\%) \\
\hline \multirow[t]{3}{*}{ Age } & $<2$ years & 85 & 32 & $117(29.5)$ \\
\hline & $2-10$ years & 135 & 51 & $186(47.0)$ \\
\hline & >10 years & 87 & 6 & $93(23.5)$ \\
\hline \multirow[t]{2}{*}{ Gender } & Female & 185 & 37 & $222(56.1)$ \\
\hline & Male & 122 & 52 & $174(43.9)$ \\
\hline \multirow[t]{2}{*}{ Reason for veterinary visit } & Check up & 81 & 64 & $145(36.6)$ \\
\hline & Illness & 226 & 25 & $251(63.4)$ \\
\hline \multirow[t]{2}{*}{ Habitat type } & Indoor & 35 & 59 & $94(23.7)$ \\
\hline & Mixed & 272 & 30 & $302(76.3)$ \\
\hline \multirow[t]{2}{*}{ Diet } & Commercial & 107 & 34 & $141(35.6)$ \\
\hline & Mixed & 200 & 55 & $255(64.4)$ \\
\hline \multirow[t]{3}{*}{ Animal antimicrobial treatments } & None & 71 & 67 & $138(34.8)$ \\
\hline & Just one & 69 & 12 & $81(20.5)$ \\
\hline & Two or more & 167 & 10 & $177(44.7)$ \\
\hline \multirow[t]{2}{*}{ Animal quinolone treatments } & Yes & 121 & 10 & $131(33.1)$ \\
\hline & No & 186 & 79 & $265(66.9)$ \\
\hline \multirow[t]{2}{*}{ Owners health professionals } & Yes & 65 & 27 & $92(23.2)$ \\
\hline & No & 242 & 62 & $304(76.8)$ \\
\hline \multirow[t]{2}{*}{ Owners antimicrobial treatments } & Yes & 162 & 25 & $187(47.2)$ \\
\hline & No & 145 & 64 & $209(52.8)$ \\
\hline \multirow[t]{2}{*}{ Cohabitant pets } & Yes & 142 & 66 & $208(52.5)$ \\
\hline & No & 165 & 23 & $188(47.5)$ \\
\hline \multirow[t]{2}{*}{ Coprophagy habits } & Yes & 119 & 18 & $137(34.6)$ \\
\hline & No & 188 & 71 & $259(65.4)$ \\
\hline
\end{tabular}

Twenty-three dogs (29.5\%) and 15 cats (68.2\%) were healthy animals admitted for regular check-ups or prophylactic actions, whereas the remaining animals attended the hospital for clinical reasons.

\subsection{Distribution of potential risk markers among E. coli isolates}

The distribution of potential risk markers among the E. coli isolates is displayed in Table 3 . The largest numbers of isolates were obtained from pets owned by non-health professionals $(n=304 ; 76.8 \%)$ and animals with outdoor access $(n=302 ; 76.3 \%)$. The characteristics that were associated with a small proportion of isolates were having only one antimicrobial treatment $(n=81 ; 20.5 \%)$, age greater than 10 years $(n=93 ; 23.5 \%)$ and living indoors $(n=94 ; 23.7 \%)$.

\subsection{Antimicrobial resistance and potential risk markers}

The frequencies of AMR for each potential risk marker are shown in Table 4. No isolate displayed resistance to nitrofurantoin or imipenem which were for this reason excluded from further statistical analyses. The AMR proportions were calculated based on all of the isolates (396). The lowest AMR rates were found in young indoor animals nourished with a commercial diet and subjected to a single previous antimicrobial treatment. In this group, there were no isolates resistant to amoxicillin-clavulanic acid and cephoxitin, and no cephoxitin-resistant isolates were found in cats or in young or indoor animals. Finally, no cefotaxime-resistant isolates were found in cats, and no gentamicin-resistant isolates were recovered from animals fed a commercial diet.

\subsection{Results of the multilevel univariable analysis}

Table 5 displays the results of the multilevel univariable analysis. Only the odds ratios (ORs) for the variables and categories in which the $p$ value was less than 0.15 are shown. There were no significant associations between the risk markers assessed and AMR for amikacin and tobramycin; therefore, these were not included in the subsequent multilevel multivariable analysis. For this reason only 15 AM were tested in this analysis.

\subsection{Results of the multilevel multivariable analysis}

The final models were obtained through a multilevel multivariate analysis after manual backwards and forwards variable selection. The variables retained after adjustment are the ones which remained significant at $p<0.05$ and are presented in Table 6 . There were no interactions among variables for none of the 15 AM tested.

In this analysis, ampicillin was the antimicrobial agent positively associated with the highest number of markers (5 of 12 markers), including species (canine), gender (male), previous quinolone treatment, having owners that were health professionals and coprophagic habits. Resistances to amoxicillin-clavulanic acid and chloramphenicol were both significantly associated with three and two different markers, respectively. Resistances to ciprofloxacin, streptomycin and trimethoprim-sulfamethoxazole showed an 
Table 4

Percentage of antimicrobial resistance distributed by categories of potential risk markers.

\begin{tabular}{|c|c|c|c|c|c|c|c|c|c|c|c|c|c|c|c|}
\hline Risk markers & AMP & AMC & CEF & FOX & CAZ & CTX & NAL & CIP & GEN & TET & STR & SXT & $\mathrm{CHL}$ & KAN & ATM \\
\hline Species canine & 58.3 & 15.0 & 53.1 & 7.5 & 14.7 & 18.9 & 39.1 & 35.2 & 7.2 & 47.6 & 45.6 & 39.4 & 20.2 & 14.0 & 20.8 \\
\hline Species feline & 27.0 & 2.2 & 24.7 & 0.0 & 10.1 & 0.0 & 24.7 & 10.1 & 1.1 & 37.1 & 36.0 & 25.8 & 11.2 & 13.5 & 6.7 \\
\hline Age: <2 years & 47.0 & 2.6 & 37.6 & 0.0 & 11.1 & 14.5 & 25.6 & 25.6 & 1.7 & 35.9 & 35.0 & 30.8 & 12.8 & 6.0 & 16.2 \\
\hline Age: $2-10$ years & 44.6 & 9.1 & 40.3 & 5.4 & 10.2 & 12.4 & 35.5 & 28.5 & 5.4 & 45.2 & 44.6 & 34.9 & 18.8 & 17.2 & 14.0 \\
\hline Age: >10 years & 69.9 & 30.1 & 71.0 & 14.0 & 23.7 & 19.4 & 49.5 & 36.6 & 11.8 & 57.0 & 51.6 & 46.2 & 23.7 & 17.2 & 26.9 \\
\hline Gender female & 45.0 & 10.8 & 44.1 & 4.1 & 11.3 & 14.4 & 34.2 & 27.5 & 6.3 & 45.5 & 40.1 & 36.0 & 11.3 & 14.9 & 16.7 \\
\hline Gender male & 59.2 & 13.8 & 50.0 & 8.0 & 16.7 & 14.9 & 37.9 & 32.2 & 5.2 & 44.8 & 47.7 & 36.8 & 27.0 & 12.6 & 19.0 \\
\hline Reason: check up & 38.6 & 4.1 & 30.3 & 1.4 & 4.8 & 3.4 & 23.4 & 17.2 & 1.4 & 34.5 & 30.3 & 33.1 & 9.7 & 11.7 & 6.9 \\
\hline Reason: illness & 58.6 & 16.7 & 56.2 & 8.4 & 18.7 & 21.1 & 43.0 & 36.7 & 8.4 & 51.4 & 51.0 & 38.2 & 23.1 & 15.1 & 23.9 \\
\hline Habitat: indoor & 37.2 & 2.1 & 33.0 & 0.0 & 13.8 & 4.3 & 39.4 & 25.5 & 2.1 & 48.9 & 41.5 & 23.4 & 20.2 & 17.0 & 11.7 \\
\hline Habitat: mixed & 55.6 & 15.2 & 51.0 & 7.6 & 13.6 & 17.9 & 34.8 & 30.8 & 7.0 & 44.0 & 44.0 & 40.4 & 17.5 & 12.9 & 19.5 \\
\hline Diet: commercial & 44.7 & 12.8 & 46.1 & 9.2 & 16.3 & 11.3 & 34.0 & 24.1 & 0.0 & 44.7 & 34.0 & 34.0 & 13.5 & 5.7 & 16.3 \\
\hline Diet: mixed & 54.9 & 11.8 & 47.1 & 3.9 & 12.2 & 16.5 & 36.9 & 32.5 & 9.0 & 45.5 & 48.6 & 37.6 & 20.8 & 18.4 & 18.4 \\
\hline AM Tx: none & 40.6 & 5.1 & 32.6 & 0.7 & 4.3 & 6.5 & 18.1 & 13.8 & 3.6 & 31.2 & 29.0 & 29.7 & 10.9 & 11.6 & 6.5 \\
\hline AM Tx: one & 45.7 & 0.0 & 40.7 & 0.0 & 11.1 & 17.3 & 44.4 & 44.4 & 7.4 & 48.1 & 43.2 & 38.3 & 19.8 & 16.0 & 22.2 \\
\hline AM Tx: 2 or + & 62.1 & 23.2 & 60.5 & 12.4 & 22.0 & 19.8 & 45.8 & 35.0 & 6.8 & 54.8 & 54.8 & 40.7 & 23.2 & 14.7 & 24.3 \\
\hline Quinolone Tx: yes & 77.1 & 19.1 & 69.5 & 12.2 & 32.8 & 33.6 & 67.2 & 56.5 & 13.0 & 63.4 & 64.9 & 51.1 & 34.4 & 21.4 & 42.0 \\
\hline Quinolone Tx: no & 38.5 & 8.7 & 35.5 & 2.6 & 4.2 & 5.3 & 20.4 & 16.2 & 2.3 & 36.2 & 32.8 & 29.1 & 10.2 & 10.2 & 5.7 \\
\hline O. Prof.: health prof. & 65.2 & 23.9 & 55.4 & 6.5 & 7.6 & 7.6 & 37.0 & 34.8 & 1.1 & 56.5 & 55.4 & 53.3 & 19.6 & 10.9 & 10.9 \\
\hline O. Prof.: others & 47.0 & 8.6 & 44.1 & 5.6 & 15.5 & 16.8 & 35.5 & 28.0 & 7.2 & 41.8 & 39.8 & 31.2 & 17.8 & 14.8 & 19.7 \\
\hline O. AM Tx: yes & 54.5 & 15.0 & 50.8 & 6.4 & 14.4 & 18.7 & 43.9 & 36.9 & 9.1 & 47.6 & 50.8 & 40.1 & 19.8 & 16.0 & 19.3 \\
\hline O. AM Tx: no & 48.3 & 9.6 & 43.1 & 5.3 & 12.9 & 11.0 & 28.7 & 23.0 & 2.9 & 43.1 & 36.8 & 33.0 & 16.7 & 12.0 & 16.3 \\
\hline Cohabit. pets: yes & 51.4 & 15.9 & 45.7 & 7.7 & 13.0 & 17.8 & 38.5 & 34.1 & 7.7 & 46.6 & 46.6 & 43.3 & 20.2 & 15.4 & 21.2 \\
\hline Cohabit. pets: no & 51.1 & 8.0 & 47.9 & 3.7 & 14.4 & 11.2 & 33.0 & 24.5 & 3.7 & 43.6 & 39.9 & 28.7 & 16.0 & 12.2 & 13.8 \\
\hline Coprophagy: yes & 67.9 & 26.3 & 56.9 & 13.1 & 19.7 & 22.6 & 48.9 & 42.3 & 10.9 & 57.7 & 57.7 & 54.0 & 23.4 & 16.8 & 27.7 \\
\hline Coprophagy: no & 42.5 & 4.6 & 41.3 & 1.9 & 10.4 & 10.4 & 29.0 & 22.8 & 3.1 & 38.6 & 35.9 & 27.0 & 15.4 & 12.4 & 12.4 \\
\hline
\end{tabular}

Legend: AM - antimicrobial; TX - treatment; O. - owner; Prof. - professional; Cohabit. - cohabitant; AMP - ampicillin; AMC - amoxicillin-clavulanic acid; CEF - cephalothin; FOX - cephoxitin; CAZ - ceftazidime; CTX - cefotaxime; NAL - nalidixic acid; CIP - ciprofloxacin; GEN - gentamicin; NIT - nitrofurantoin; TET - tetracycline; STR - streptomycin; SXT - trimethoprim-sulfamethoxazole; CHL - chloramphenicol; KAN - kanamycin; IPM - imipenem; ATM aztreonam.

association with two similar markers, namely previous quinolone treatment and coprophagic habits, whereas AMR to cephalothin, ceftazidime, cefotaxime, nalidixic acid, gentamycin, tetracycline, and aztreonam retained one significant association (previous quinolone treatment). Finally, cephoxitin resistance was associated with coprophagy, and kanamycin resistance was positively associated with a mixed diet.

Previous quinolone treatments and coprophagic habits were significantly related to AMR for 12 and 6 of the 15 antimicrobial agents tested, respectively. According to the model, pets that received quinolone treatments have a significantly higher risk of colonization by $E$. coli resistant to ceftazidime (OR 16.78 (2.33-120.74)), cefotaxime (OR 22.01 (13.15-154.01)), nalidixic acid (OR 13.51 (3.83-47.61)) and aztreonam (OR 19.18 (3.67-100.14)). Animals with coprophagic habits are at a higher risk of harboring $E$. coli isolates resistant to amoxicillin-clavulanic acid (OR 10.35 (2.34-45.76)) and cephoxitin (OR 11.21 (1.26-99.64)) (Table 6). Overall, the risk markers significantly associated with AMR were the following: (i) previous treatment with quinolones (12 of 15) and (ii) coprophagic habits (6 of 15). The following variables were only sporadically associated with AMR to some antimicrobials: (i) canine species (ampicillin); (ii) male gender (ampicillin and chloramphenicol); (iii) illness (amoxicillin-clavulanic acid and chloramphenicol); (iv) mixed diet (kanamycin) and (v) health professional owners (ampicillin and amoxicillin-clavulanic acid).

\section{Discussion}

Given the remarkable increase in AMR worldwide and the enormous difficulties and unsuccessful strategies to prevent resistance, it is important to identify resistance risk factors. The present study was designed to assess the prevalence of AMR in enteric E. coli isolated from domestic cats and dogs in Porto, Portugal and to identify the potential risk markers for the presence of AMR in those isolates. This was accomplished with a GLMM because of the multilevel structure of the data. The inspection of the address information of the owners enrolled in the study showed no evidence of geographical clustering.

The proportions of AMR observed against ampicillin, cephalothin, tetracycline, streptomycin, trimethoprimsulfamethoxazole, nalidixic acid, and ciprofloxacin were higher than previously reported (Costa et al., 2008; Murphy et al., 2009; Leonard et al., 2012). According to the categories proposed by the EFSA (EFSA and ECDPC, 2013), $36.9 \%$ of the AMs tested were in the high or very high resistance groups (Table 1 ). Interestingly, none of the AMs tested were classified in the extremely high 
Table 5

Risk markers for the antimicrobial resistance of $E$. coli isolates from the univariable multilevel analysis.

\begin{tabular}{|c|c|c|c|c|c|c|c|c|c|c|c|c|c|c|c|}
\hline Risk markers & $\begin{array}{l}\text { AMP } \\
\text { OR }\end{array}$ & $\begin{array}{l}\text { AMC } \\
\text { OR }\end{array}$ & $\begin{array}{l}\text { CEF } \\
\text { OR }\end{array}$ & $\begin{array}{l}\text { FOX } \\
\text { OR }\end{array}$ & $\begin{array}{l}\text { CAZ } \\
\text { OR }\end{array}$ & $\begin{array}{l}\text { CTX } \\
\text { OR }\end{array}$ & $\begin{array}{l}\text { NAL } \\
\text { OR }\end{array}$ & $\begin{array}{l}\text { CIP } \\
\text { OR }\end{array}$ & $\begin{array}{l}\text { GEN } \\
\text { OR }\end{array}$ & $\begin{array}{l}\text { TET } \\
\text { OR }\end{array}$ & $\begin{array}{l}\text { STR } \\
\text { OR }\end{array}$ & $\begin{array}{l}\text { SXT } \\
\text { OR }\end{array}$ & $\begin{array}{l}\text { CHL } \\
\text { OR }\end{array}$ & $\begin{array}{l}\text { KAN } \\
\text { OR }\end{array}$ & $\begin{array}{l}\text { ATM } \\
\text { OR }\end{array}$ \\
\hline $\begin{array}{l}\text { Species canine } \\
\text { Species feline }\end{array}$ & $6.63^{* *}$ & 7.08 & $5.37^{* *}$ & & & & 2.43 & $5.83^{*}$ & & & & & & & 4.76 \\
\hline $\begin{array}{l}\text { Age: }<2 \text { years } \\
\text { Age: } 2-10 \text { years } \\
\text { Age: }>10 \text { years }\end{array}$ & $\begin{array}{l}0.3 \\
0.24^{*}\end{array}$ & $\begin{array}{l}0.06^{* *} \\
0.17^{*}\end{array}$ & $\begin{array}{l}0.14^{* *} \\
0.16^{* *}\end{array}$ & & $\begin{array}{l}0.42 \\
0.28\end{array}$ & & $\begin{array}{l}0.26 \\
0.46\end{array}$ & $\begin{array}{l}0.53 \\
0.61\end{array}$ & & $\begin{array}{l}0.29 \\
0.52\end{array}$ & & & & & \\
\hline $\begin{array}{l}\text { Gender female } \\
\text { Gender male }\end{array}$ & 0.4 & & & & & & & & & & & & $0.25^{*}$ & & \\
\hline $\begin{array}{l}\text { Reason: check up } \\
\text { Reason: ilness }\end{array}$ & $0.30^{*}$ & $0.22^{*}$ & $0.23^{* *}$ & & 0.26 & $0.14^{*}$ & $0.35^{*}$ & $0.32^{*}$ & & $0.36^{*}$ & $0.30^{*}$ & & 0.36 & & $0.23^{*}$ \\
\hline $\begin{array}{l}\text { Habitat: indoor } \\
\text { Habitat: mixed }\end{array}$ & 0.31 & 0.13 & 0.33 & & & 0.16 & & & & & & 0.38 & & & \\
\hline $\begin{array}{l}\text { Diet: commercial } \\
\text { Diet: mixed }\end{array}$ & & & & & & & & & & & 0.42 & & & $0.24 *$ & \\
\hline $\begin{array}{l}\text { AM Tx: none } \\
\text { AM Tx: one } \\
\text { AM Tx: } 2 \text { or }+\end{array}$ & $\begin{array}{l}0.30^{*} \\
0.35\end{array}$ & $\begin{array}{l}0.19^{*} \\
0.00\end{array}$ & $\begin{array}{l}0.21^{* *} \\
0.31\end{array}$ & $\begin{array}{l}0.10 \\
0.00\end{array}$ & $\begin{array}{l}0.17^{*} \\
0.41\end{array}$ & $\begin{array}{l}0.26 \\
0.78\end{array}$ & $\begin{array}{l}0.20^{* *} \\
0.89\end{array}$ & $\begin{array}{l}0.25^{*} \\
1.59\end{array}$ & & $\begin{array}{l}0.24^{*} \\
0.66\end{array}$ & $\begin{array}{l}0.22^{* *} \\
0.475\end{array}$ & & & & $\begin{array}{l}0.19^{*} \\
0.97\end{array}$ \\
\hline $\begin{array}{l}\text { Quinolone Tx: yes } \\
\text { Quinolone Tx: no }\end{array}$ & $10.01^{* * *}$ & & $7.71^{* * * *}$ & 4.5 & $15.94^{* * *}$ & $13.84^{* * * *}$ & $14.92^{* * *}$ & $12.28^{* * *}$ & $4.73 *$ & $4.76^{* *}$ & $6.98^{* * *}$ & $3.43^{*}$ & $6.42^{* *}$ & 2.4 & $20.79^{* * *}$ \\
\hline $\begin{array}{l}\text { O. Prof.: health prof. } \\
\text { O. Prof.: others }\end{array}$ & 2.7 & $4.69^{*}$ & & & & & & & & & & & & & \\
\hline $\begin{array}{l}\text { O. AM Tx: yes } \\
\text { O. AM Tx: no }\end{array}$ & & & & & & & 2.51 & 2.23 & & & 2.65 & & & & \\
\hline \multicolumn{16}{|l|}{$\begin{array}{l}\text { Cohabit. Pets: yes } \\
\text { Cohabit. Pets: no }\end{array}$} \\
\hline $\begin{array}{l}\text { Coprophagy: yes } \\
\text { Coprophagy: no }\end{array}$ & $4.14^{* *}$ & $10.43^{* *}$ & 2.39 & $10.98^{*}$ & & 3.22 & $3.22^{*}$ & $3.61^{*}$ & 3.81 & $3.07^{*}$ & $3.79^{*}$ & $4.77^{* *}$ & & & $3.43^{*}$ \\
\hline
\end{tabular}

Risk markers for the antimicrobial resistance of $E$. coli isolates from the univariable multilevel analysis.

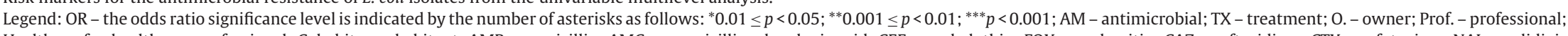

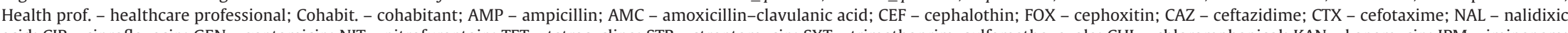

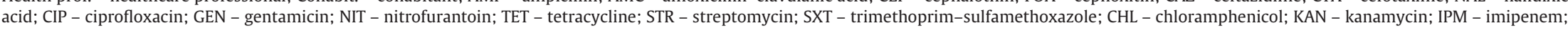
ATM - aztreonam. 
Table 6

Final results with risk markers for the antimicrobial resistance of $E$. coli isolates $(n=396)$ from the multilevel multivariable analysis.

\begin{tabular}{|c|c|c|c|c|c|}
\hline AM & Risk marker & AMR+ & OR & $\mathrm{CI}$ & $p$ Value \\
\hline \multirow[t]{5}{*}{ AMP } & Species canine & 179 & 5.16 & $1.49-17.85$ & 0.010 \\
\hline & Gender female & 100 & 0.35 & $0.13-0.95$ & 0.039 \\
\hline & Previous quinolone tx. & 101 & 6.02 & $2.02-17.89$ & 0.001 \\
\hline & O. Health prof. & 60 & 3.95 & $1.23-12.70$ & 0.021 \\
\hline & Coprophagy & 93 & 2.80 & $1.02-7.67$ & 0.045 \\
\hline \multirow[t]{3}{*}{ AMC } & Reason Check up & 6 & 0.16 & $0.03-0.98$ & 0.047 \\
\hline & O. Health prof. & 22 & 6.41 & $1.34-29.88$ & 0.018 \\
\hline & Coprophagy & 36 & 10.35 & $2.34-45.76$ & 0.002 \\
\hline CEF & Previous quinolone tx. & 91 & 4.68 & $1.15-19.10$ & 0.032 \\
\hline FOX & Coprophagy & 18 & 11.21 & $1.26-99.64$ & 0.030 \\
\hline CAZ & Previous quinolone tx. & 43 & 16.78 & $2.33-120.74$ & 0.005 \\
\hline CTX & Previous quinolone tx. & 44 & 22.01 & $13.15-154.01$ & 0.002 \\
\hline NAL & Previous quinolone tx. & 88 & 13.51 & $3.83-4.61$ & 0.000 \\
\hline \multirow[t]{2}{*}{ CIP } & Previous quinolone tx. & 74 & 9.05 & $2.62-31.30$ & 0.001 \\
\hline & Coprophagy & 58 & 3.12 & $1.05-9.29$ & 0.040 \\
\hline GEN & Previous quinolone tx & 17 & 4.73 & $1.02-22.81$ & 0.049 \\
\hline TET & Previous quinolone tx & 83 & 4.20 & $1.46-12.07$ & 0.008 \\
\hline \multirow[t]{2}{*}{ STR } & Previous quinolone $\mathrm{tx}$ & 85 & 4.55 & $1.29-16.09$ & 0.019 \\
\hline & Coprophagy & 79 & 3.20 & $1.09-9.45$ & 0.035 \\
\hline \multirow[t]{2}{*}{ SXT } & Previous quinolone tx & 67 & 2.87 & $1.05-7.78$ & 0.039 \\
\hline & Coprophagy & 74 & 3.73 & $1.32-10.48$ & 0.013 \\
\hline \multirow[t]{2}{*}{$\mathrm{CHL}$} & Gender female & 25 & 0.28 & $0.09-0.93$ & 0.038 \\
\hline & Previous quinolone tx & 45 & 4.79 & $1.33-17.25$ & 0.017 \\
\hline KAN & Diet commercial & 8 & 0.22 & $0.058-0.812$ & 0.023 \\
\hline ATM & Previous quinolone tx & 55 & 19.18 & $3.67-100.14$ & 0.000 \\
\hline
\end{tabular}

Legend: OR - odds ratio; CI - confidence interval; tx - treatment; O. - owner; Prof. - professional; Health prof. - healthcare professional; AMP - ampicillin; AMC - amoxicillin-clavulanic acid; CEF - cephalothin; FOX - cephoxitin; CAZ - ceftazidime; CTX - cefotaxime; NAL - nalidixic acid; CIP - ciprofloxacin; GEN - gentamicin; NIT - nitrofurantoin; TET - tetracycline; STR - streptomycin; SXT - trimethoprim-sulfamethoxazole; CHL - chloramphenicol; KAN kanamycin; IPM - imipenem; ATM - aztreonam.

category. In a study comprising fecal samples from 565 stray and 312 hospitalized dogs, Nam et al. (2010) reported generally higher AMR rates. However, according to Korea Health Products Association data regarding the amount of antimicrobials used in pets, this conclusion was previously reached by the authors, who hypothesized that resistance is related to the categories and elevated antimicrobial consumption rates in the country.

Because no antimicrobial was administered to the animals enrolled in the present study during the four months prior to sampling, our results are consistent with the hypothesis that the reversibility of resistance in the absence of AM is a slow process, most likely due to compensatory evolution and cost-free resistance mechanisms (Andersson and Hughes, 2009). Although the Porto city area follows the urban trend of longer pet longevity, better veterinary care and widespread use of antibiotics in companion animal treatments, there is no evidence that these characteristics are different from those of other studied areas. It has been demonstrated, however, that the Porto region suffers from a high level of environmental contamination with antimicrobial resistance determinants (Novais et al., 2005; Simões et al., 2010; Flores et al., 2013); therefore, the acquisition of resistance may be multifactorial (Martínez, 2012), and environment contamination exposure may also contribute to the high AMR rates.

Two variables influencing $E$. coli AMR deserve special attention because of their relationship with resistance to several antimicrobials. These risk markers are prior quinolone treatment and coprophagic habits, which are discussed in detail below.
During coprophagic behavior, the animal ingests gut microflora, including multidrug-resistant E. coli strains, from himself, which means a re-inoculation (autocoprophagy), or from other animals (allocoprophagy). Those strains, particularly those from autocoprophagy, are expected to be adapted for prolonged colonization. Furthermore, feces from animals undergoing AM treatments, particularly with poor oral bioavailability, may contain residual concentrations of the drug that are high enough to pressure the emergence and dissemination of AMR (Thaller et al., 2010; Toutain et al., 2010). Finally, several studies have shown that there is a high level of horizontal gene transfer (HGT) within the intestine and that its warm and nutrient-rich environment makes it an ideal location for such a phenomenon (Lester et al., 2006; Hammerum and Heuer, 2009; Jakobsson et al., 2010).

Among the 15 studied antimicrobials, 12 had resistance rates related to previous quinolone treatments. Previous quinolone exposure had previously been indicated as a risk marker for the emergence of AMR in E. coli isolated from food animals (Moniri and Dastehgoli, 2005) and humans (Cheong et al., 2001; McDonald et al., 2001; Lastours et al., 2010). This occurrence has been explained by the possible association of multiple antimicrobial resistance genes on mobile genetic elements (Moreno et al., 2008; Strahilevitz et al., 2009). Additionally, a strong association of plasmid-mediated quinolone resistance (PMQR) determinants with extended-spectrum- $\beta$-lactamases (ESBLs) or AmpC-type- $\beta$-lactamases has been reported (Moreno et al., 2008; Hammerum and Heuer, 2009; Strahilevitz et al., 2009; Rawat and Nair, 2010). These two types of resistance genes are often co-localized on the same plasmid, 
along with genetic determinants of other antimicrobial agents, such as aminoglycosides, trimethoprim, sulfonamides, tetracyclines and chloramphenicol (Jacoby, 2009; Strahilevitz et al., 2009; Rawat and Nair, 2010; Zhao et al., 2010).

The significantly higher risk for ampicillin and amoxicillin-clavulanic acid resistance in pets whose owners are healthcare workers may be due to the combination of two driving forces. First, because genetic determinants for aminopenicillin resistance often circulate among medical staff and facilities, pets are at increased risk of acquiring antimicrobial-resistant $E$. coli from these owners (Hammerum and Heuer, 2009; Kalter et al., 2010; Martins da Costa et al., 2013). Second, the transfer of specific resistance determinants to endogenous strains may be significantly enhanced by recurrent exposure to the most prescribed oral antimicrobial drug in Portugal, namely amoxicillin-clavulanic acid (DGAV, 2011).

At the univariable level, the model used in the present study showed a clear and positive association between previous antimicrobial exposure and AMR. After our results (Table 5), it can be hypothesized that animals with a relevant clinical background (pe, previously exposed to an antimicrobial treatment) harbor more resistant E. coli isolates. This was also reported by Moyaert et al. (2006), whose work with hospitalized animals showed frequencies of AMR similar to the rates found in our study, which included nearly two thirds (62\%) of patients with chronic conditions recurrently exposed to antimicrobial treatments. A similar effect was observed with the age variable for the $\beta$-lactamics ampicillin, amoxicillin-clavulanic acid and cephalothin. Therefore, younger and healthy animals carry less resistant $E$. coli strains, which may be related to fewer opportunities of contact with antimicrobials. This, added to fewer cases of coprophagic habits, is also a plausible explanation for the lower prevalence of antibiotic resistance in cats compared to dogs, which exhibit a 5.16-fold higher odds of carrying ampicillin-resistant $E$. coli.

Finally, at the univariable level, the difference in the risk for contamination with multidrug-resistant $E$. coli in outdoor compared with indoor animals was not statistically significant. However, as shown by Boothe (2012), male gender was considered a risk marker for resistance to ampicillin and chloramphenicol.

The limitations of the study are primarily related to the number of pets enrolled, which was not calculated in advance because the purpose was to include the most animals possible given the time and resources available. However, because the selection process was random, the pets studied represent the hospital population and ensure an important factor of external validity. The statistical analysis provides the necessary significance to assess the risk markers, taking account of the sample size. The microbiological isolation, identification and antimicrobial resistance determination followed internal quality control procedures to ensure reproducibility (consolidated methods performed by trained personnel) and accuracy (quality control of isolation and antimicrobial resistance media and internal control strains with known resistance patterns).

In the questionnaire, the section "Previous antimicrobial treatments" was designed to record the antimicrobials commercial name. However, after the data analyses, it came clear that the high variety of antimicrobial drugs used by the owners hindered the statistical study. So, it was decided to merge this data into a categorical variable with three levels, as follows: "none", "just one" and "two or more" antimicrobial treatments. To overcome the problem of memory bias, the authors decided to draw the attention of the owner on quinolones, as a group of particular importance to the study, by presenting them a list of commercial names from which they would recognize a particular one if it was the case. Despite the fact that topical and/or ear treatments information has also been collected with the questionnaire, considering that these treatments were scarce and the microorganisms studied were commensal from feces, the authors decided not to include such data into the analysis. Even though the evaluation of its indirect influence at the antimicrobial resistance has not been possible, it could be interesting.

\section{Conclusion}

The present survey showed an increased risk of AMR in enteric E. coli strains from pets with a record of previous quinolone treatments, which is consistent with the results of several other studies in different animal species. The pets displaying coprophagic behavior showed an increased risk of AMR in enteric E. coli strains, which highlight the role of veterinarians in alerting and educating pet owners in order to prevent and limit such behavior. Other markers, such as gender, species, and reason for check-up, were statistically significant for a small number of antimicrobials. Additional studies of AMR risk markers are necessary.

\section{Conflict of interest statement}

The authors declare that they have no conflicts of interest.

\section{Acknowledgements}

The authors thank Bruno Ramos and all members of the UPVET team for their collaboration in this study. Finally, we also thank Lucinda Bessa for reviewing the final version of the manuscript.

\section{Appendix A. Supplementary data}

Supplementary data associated with this article can be found, in the online version, at http://dx.doi.org/10.1016/ j.prevetmed.2014.09.008.

\section{References}

Aarestrup, F.M., 2004. Monitoring of antimicrobial resistance among food animals: principles and limitations. J. Vet. Med. 51, 380-388.

Ahmed, M.O., Williams, N.J., Clegg, P.D., van Velkinburgh, J.C., Baptiste, K.E., Bennett, M., 2012. Analysis of risk factors associated with antibioticresistant Escherichia coli. Microb. Drug Resist. 18, 161-168.

Akwar, T.H., Poppe, C., Wilson, J., Reid-Smith, R.J., Dyck, M., Waddington, J., Shang, D., Dassie, N., McEwen, S.A., 2007. Risk factors for antimicrobial 
resistance among fecal Escherichia coli from residents on forty-three swine farms. Microb. Drug Resist. 13, 69-76.

Andersson, D.I., Hughes, D., 2009. Antibiotic resistance and its cost: is it possible to reverse resistance? Nat. Rev. Microbiol. 8, 260-271.

Boothe, D.M., 2012. Antimicrobial Resistance. Small Animal Clinical Pharmacology and Therapeutics, 2nd ed. Elsevier Saunders, St. Louis, Missouri, USA.

Carlet, J., Jarlier, V., Harbarth, S., Voss, A., Goossens, H., Pittet, D., 2012. Participants of the 3rd World Healthcare-Associated Infections Forum Ready for a world without antibiotics? The Pensières Antibiotic Resistance Call to Action. Antimicrob. Resist. Infect. Control. 1, 11.

CDC - Centers for Disease Control and Prevention, 2013. Antibiotic Resistance Threats in the United States, 2013, Available at: http://www.cdc.gov/drugresistance/threatreport-2013/pdf/arthreats-2013-508.pdf</ce:inter-ref>

Cheong, H.J., Yoo, C.W., Sohn, J.W., Kim, W.J., Kim, M.J., Park, S.C., 2001. Bacteremia due to quinolone-resistant Escherichia coli in a teaching hospital in South Korea. Clin. Infect. Dis. 33, 48-53.

Clinical Laboratory Standards Institute (CLSI), 2012. Performance Standards for Antimicrobial Susceptibility Testing; Seventeenth Informational Supplement (CLSI Doc. M100-S17). CLSI, Wayne, PA.

Costa, D., Poeta, P., Saenz, Y., Coelho, A.C., Matos, M., Vinue, L., Rodrigues, J., Torres, C., 2008. Prevalence of antimicrobial resistance and resistance genes in faecal Escherichia coli isolates recovered from healthy pets. Vet. Microbiol. 127, 97-105.

Direcção Geral de Alimentação e Veterinária (DGAV), 2011. Relatório Nacional de Monitorização do Consumo de Antimicrobianos em Animais em Portugal., pp. 1-19.

European Antibiotic Awareness Day (EAAD), 2013. http://www.ecdc europa.eu/en/press/Press\%20Releases/antimicrobial-resistance-ratescarbapenem-resistant-infections-continue-to-increase-in-Europe.pdf

European Food Safety Authority (EFSA), European Centre for Disease Prevention and Control (ECDPC), 2013. The European Union Summary Report on antimicrobial resistance in zoonotic and indicator bacteria from humans, animals and food in 2011. EFSA J. 11, 3196 (359 pp.).

Elseviers, M.M., Ferech, M., Vander Stichele, R.H., Goossens, H. Project Group, E.S.A.C., 2007. Antibiotic use in ambulatory care in Europe (ESAC data 1997-2002): trends, regional differences, and seasonal fluctuations. Pharmacoepidemiol. Drug Saf. 16, $115-123$.

Flores, C.E., Loureiro, L., Bessa, L.J., Martins da Costa, P., 2013. Presence of Multidrug-Resistant E. coli, Enterococcus spp. and Salmonella spp. in Lakes and Fountains of Porto, Portugal. J. Water Resour. Prot. 5 , 1117-1126.

Goossens, H., Ferech, M., Stichele, R.V., Elseviers, M., 2005. Outpatient antibiotic use in Europe and association with resistance: a crossnational database study. Lancet 365, 579-587.

Hammerum, A., Heuer, O., 2009. Human health hazards from antimicrobial-resistant Escherichia coli of animal origin. Clin. Infect. Dis. 48, 916-921.

Jacoby, G.A., 2009. AmpC- $\beta$-lactamases. Clin. Microbiol. Rev. 22, 161-182.

Jakobsson, H.E., Jernberg, C., Andersson, A.F., Sjölund-Karlsson, M., Jansson, J.K., Engstrand, L., 2010. Short-term antibiotic treatment has differing long-term impacts on the human throat and gut microbiome. PLoS ONE 5, e9836.

Jernberg, C., Löfmark, S., Edlund, C., Jansson, J.K., 2010. Long-term impacts of antibiotic exposure on the human intestinal microbiota. Microbiology 156, 3216-3223.

Kalter, H.D., Gilman, R.H., Moulton, L.H., Cullotta, A.R., Cabrera, L., Velapatiño, B., 2010. Risk factors for antibiotic-resistant Escherichia coli carriage in young children in Peru: community-based cross-sectional prevalence study. Am. J. Trop. Med. Hyg. 82, 879-888.

Kaper, J.B., Nataro, J.P., Mobley, H.L.T., 2004. Pathogenic Escherichia coli. Nat. Rev. Microbiol. 2, 123-140.

Kearns, D.B., 2010. A field guide to bacterial swarming motility. Nat. Rev. Microbiol. 8, 634-644.

Lastours, V., Chau, F., Tubach, F., Pasquet, B., Ruppé, E., Fantin, B., 2010. Independent behavior of commensal flora for carriage of fluoroquinolone-resistant bacteria in patients at admission. Antimicrob. Agents Chemother. 54, 5193-5200

Leonard, E., Pearl, D., Finley, R., Janecko, N., Reid-Smith, R., Peregrine, A., Weese, J., 2012. Comparison of antimicrobial resistance patterns of Salmonella spp. and Escherichia coli recovered from pet dogs from volunteer households in Ontario (2005-2006). J. Antimicrob. Chemother. 67, 174-181.

Lester, C.H., Frimodt-Moller, N., Sorensen, T.L., Monnet, D.L., Hammerum, A.M., 2006. In vivo transfer of the vanA resistance gene from an
Enterococcus faecium isolate of animal origin to an E. faecium isolate of human origin in the intestines of human volunteers. Antimicrob. Agents Chemother. 50, 596-599.

Lietzau, S., Raum, E., von Baum, H., Marre, R., Brenner, H., 2007. Household contacts were key factor for children's colonization with resistant Escherichia coli in community setting. J. Clin. Epidemiol. 60, 1149-1155.

Lloyd, D.H., 2007. Reservoirs of antimicrobial resistance in pet animals. Clin. Infect. Dis. 45, S148-S152.

Magiorakos, A.P., Srinivasan, A., Carey, R.B., Carmeli, Y., Falagas, M.E., Giske, C.G., Harbarth, S., Hindler, J.F., Kahlmeter, G., Olsson-Liljequist, B., Paterson, D.L., Rice, L.B., Stelling, J., Struelens, M.J., Vatopoulos, A., Weber, J.T., Monnet, D.L., 2012. Multidrug-resistant, extensively drugresistant and pandrug-resistant bacteria: an international expert proposal for interim standard definitions for acquired resistance. Clin. Microbiol. Infect. 18, 268-281.

Martínez, J., 2012. Natural antibiotic resistance and contamination by antibiotic resistance determinants: the two ages in the evolution of resistance to antimicrobials. Front. Microbiol. 3, 1-3.

Martins, L.R.L., Pina, S.M.R., Rocha, R.L., de Matos, A.J.F., Rodigues, P., da Costa, P.M.R., 2013. Common phenotypic and genotypic antimicrobial resistance patterns found in a case study of multiresistant $E$. coli from cohabitant pets, humans, and household surfaces. J. Environ. Health 75, 74-81.

Martins da Costa, P., Loureiro, L., Matos, A.J.F., 2013. Transfer of multidrug-resistant bacteria between intermingled ecological niches: the interface betweens humans, animals and the environment. Int. J. Environ. Res. Public Health 10, 278-294.

McDonald, L.C., Chen, F.J., Lo, H.J., Yin, H.C., Lu, P.L., Huang, C.H., Chen, P., Lauderdale, T.L., Ho, M., 2001. Emergence of reduced susceptibility and resistance to fluoroquinolones in Escherichia coli in Taiwan and contributions of distinct selective pressures. Antimicrob. Agents Chemother. 45, 3084-3091.

Moniri, R., Dastehgoli, K., 2005. Fluoroquinolone-resistant Escherichia coli isolated from healthy broilers with previous exposure to fluoroquinolones: is there a link? Microb. Ecol. Health Dis. 17, 69-74.

Moreno, A., Bello, H., Guggiana, D., Dominguez, M., Gonzalez, G., 2008. Extended-spectrum beta-lactamases belonging to CTX-M group produced by Escherichia coli strains isolated from companion animals treated with enrofloxacin. Vet. Microbiol. 129, 203-208.

Moyaert, H., De Graef, E.M., Haesebrouck, F., Decostere, A., 2006. Acquired antimicrobial resistance in the intestinal microbiota of diverse cat populations. Res. Vet. Sci. 81, 1-7.

Murphy, C., Richard, J., Reid-Smith, Prescott, J.F., Bonnett, B.N., Poppe, C., Boerlin, P., Weese, J.S., Janecko, N., McEwen, S.A., 2009. Occurrence of antimicrobial resistant bacteria in healthy dogs and cats presented to private veterinary hospitals in southern Ontario: a preliminary study. Can. Vet. J. 50, 1047-1053.

Nam, H.M., Lee, H.S., Byun, J.W., Yoon, S.S., Jung, S.C., Joo, Y.S., Lim, S.K., 2010. Prevalence of antimicrobial resistance in fecal Escherichia coli isolates from stray pet dogs and hospitalized pet dogs in Korea. Microb. Drug Resist. 16, 75-79.

Novais, C., Coque, T.M., Ferreira, H., Sousa, J.C., Peixe, L., 2005. Environmental contamination with vancomycin-resistant enterococci from hospital sewage in Portugal. Appl. Environ. Microbiol. 71, 3364-3368.

Rawat, D., Nair, D., 2010. Extended-spectrum $\beta$-lactamases in Gram negative bacteria. Glob. Infect. Dis. 2, 263-274.

Simões, R.R., Poirel, L., da Costa, P.M., Nordmann, P., 2010. Seagulls and beaches as reservoirs for Multidrug-Resistant Escherichia coli. Emerg. Infect. Dis. 16, 110-112.

Sotto, A., de Boever, C.M., Fabbro-Peray, P., Gouby, A., Sirot, D., Jourdan, J., 2001. Risk factors for antibiotic-resistant Escherichia coli isolated from hospitalized patients with urinary tract infections: a prospective study. J. Clin. Microbiol. 39, 438-444.

Strahilevitz, J., Jacoby, G.A., Hooper, D.C., Robicsek, A., 2009. Plasmidmediated quinolone resistance: a multifaceted threat. Clin. Microbiol. Rev. 22, 664-689.

Taylor, N.M., Davies, R.H., Ridley, A., Clouting, C., Wales, A.D., CliftonHadley, F.A., 2008. A survey of fluoroquinolones resistance in Escherichia coli and thermophilic Campylobacter spp. on poultry and pig farms in Great Britain. J. Appl. Microbiol. 105, 1421-1431.

Thaller, M.C., Migliore, L., Marquez, C., Tapia, W., Cedeño, V., Rossolini, G.M., Gentile, G., 2010. Tracking acquired antibiotic resistance in commensal bacteria of Galápagos land iguanas: no man, no resistance. PLoS ONE 1 (5), e8989. 
Toutain, P.L., Ferran, A., Bousquet-Mélou, A., 2010. Consequence of coprophagy on drug disposition and responses. Species Differ. pharmacokinet. Pharmacodyn. 7, 33-35.

Vigil, K.J., Javier, A.A., Aboufaycal, H., Hachem, R.Y., Reitzel, R.A., Jiang, Y., Tarrand, J.J., Chemaly, R.F., Bodey, G.P., Rolston, K.V., Raad, I., 2009. Multidrug-resistant Escherichia coli bacteremia in cancer patients. Am. J. Infect. Control. 37, 741-745.
Zhao, J., Chen, Z., Chen, S., Deng, Y., Liu, Y., Tian, W., Huang, X., Wu, C., Sun, Y., Sun, Y., Zeng, Z., Liu, J.H., 2010. Prevalence and dissemination of oqxAB in Escherichia coli isolates from animals, farmworkers, and the environment. Antimicrob. Agents Chemother. 54, 4219-4224. 